\title{
Transmission Properties of Various Split-Ring Resonator Systems
}

\author{
Koray Aydin, I. Bulu, K. Guven and Ekmel Ozbay \\ Nanotechnology Research Center and Department of Physics, Bilkent University, Bilkent, 06800 Ankara, Turkey \\ aydin@fen.bilkent.edu.tr
}

\begin{abstract}
We investigated the magnetic resonance of split-ring resonators (SRR) experimentally. The dependence of the geometrical parameters on the resonance frequency of SRR is studied. We further investigated the effect of disorder on performance of SRRs.

(C)2006 Optical Society of America

OCIS codes: (120.7000) Tranmission; (260.5740) Physical Optics, resonance.
\end{abstract}

\section{Introduction}

Materials exhibiting negative values of dielectric permittivity $(\varepsilon)$ and magnetic permeability $(\mu)$ are named as lefthanded materials (LHM) and they have a negative index of refraction [1]. The left-handed pass band frequency (i.e., the negative refractive index regime) is essentially determined by the magnetic resonance frequency $\left(\omega_{m}\right)$ of periodic SRR structures [2,3]. In this study, we first investigate the effect of geometrical parameters of SRRs and different resonator designs on the magnetic resonance frequency. Our work covers the change of $\omega_{m}$ with the split width, gap between inner and outer rings, metal width, and additional capacitances. Then we move on array of SRRs and present a systematic study of the effect of disorder and misalignments on the transmission properties of SRR structures operating in the microwave regime. We focus on the disorder of SRR layers, as the $\mu(\omega)<0$ gap defines where the left handed $\left(n_{\text {eff }}<0\right)$ transmission occurs.

\section{Magnetic resonance}

A dip in the transmission spectrum of SRR structure can be attributed to the resonant nature of SRRs. Two monopole antennas are used to transmit and detect the EM waves through the single SRR unit cell. By keeping other parameters constant, we only changed the split width $(d)$ of SRRs. Figure 1(a) displays the measured transmission spectra of 4 SRR structures with different split widths. FDTD simulations are performed to check the experimental results (not shown here). The agreement is good between numerical and experimental results. Increasing the split width of inner and outer rings, results in an increase of the magnetic resonance frequency of SRR structure.
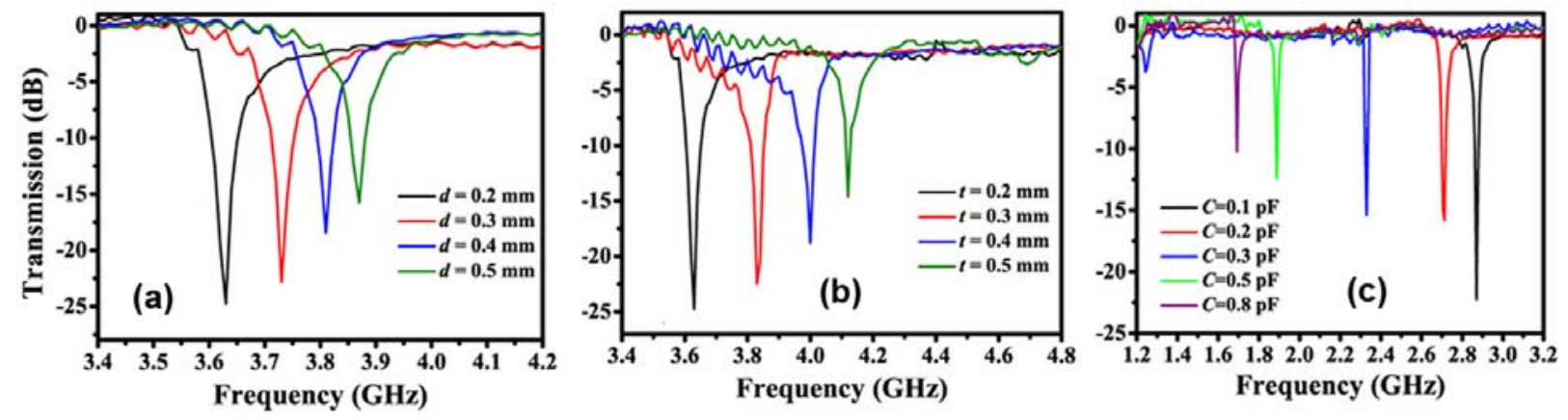

Fig. 1. Transmission spectra of SRR unit cells with (a) different split widths, (b) various gap distances and (c) different additional surface-mount capacitance values.

Then we studied the effect of gap between inner and outer rings on $\omega_{m}$ of SRR structures. The other parameters are kept constant $(d=0.2 \mathrm{~mm}, w=0.9 \mathrm{~mm}$ and $r=3.6 \mathrm{~mm})$, and only gap distance $(t)$ is varied from 0.2 to 0.6 with $0.05 \mathrm{~mm}$ steps. Figure 1(b) display the measured transmission spectra of SRRs with different gap distances. Increasing the distance between inner and outer rings, results in an increase of the magnetic resonance frequency of SRR structure.

We also changed the capacitance of the system by integrating additional capacitors to SRR structures. Surfacemount capacitors varying from $0.1 \mathrm{pF}$ to $0.8 \mathrm{pF}$ are mounted to the outer ring of SRRs in parallel. Figure 1(c) gives 


\section{JTuD44.pdf}

the measured transmission spectra of 5 different SRRs with different additional surface-mount capacitors. Without any additional capacitor, $\omega_{m}$ of the SRR structure was measured to be $3.63 \mathrm{GHz}$. Magnetic resonance frequency significantly shifts to $2.87 \mathrm{GHz}$ when $C=0.1 \mathrm{pF}$ is mounted to the outer ring. The magnetic frequency can be tuned to as low as $1.63 \mathrm{GHz}$ by using a $C=0.8 \mathrm{pF}$ capacitor. By mounting additional capacitors to the system we significantly increase the total capacitance.

\section{Effect of disorder on magnetic resonance band gap of SRRs}

SRR units are arranged periodically to obtain a negative permeability medium. The periodic SRR structure is made of $N_{x}=10, N_{y}=15$, and $N_{z}=24$ unit cells, with lattice spacings $a_{x}=a_{y}=8.8$, and $a_{z}=6.5 \mathrm{~mm}$. Periodic and ordered structure has a magnetic resonance band gap between $3.55-4.10 \mathrm{GHz}$ (blue line in Fig. 2). Then we introduced intra-plane disorders into periodic SRR system. Here we assigned randomness to the positions of SRRs along the $x$ and $y$ directions within a board. Each SRR on the board with lattice point, $\overrightarrow{\mathbf{r}}_{n}$, where $\overrightarrow{\mathbf{r}}=x \hat{\mathbf{i}}+y \hat{\mathbf{j}}$ is displaced with $\overrightarrow{\mathbf{r}}_{n} \pm \overrightarrow{\boldsymbol{\delta}}_{r}$. Therefore the degree of disorder can be changed by varying the randomness parameter, $\overrightarrow{\boldsymbol{\delta}}_{r}$. We consider two cases: (a) $\left|\overrightarrow{\boldsymbol{\delta}}_{r}\right| \leq a / 9$, and (b) $\left|\overrightarrow{\boldsymbol{\delta}}_{r}\right| \leq a / 5$, which are displayed schematically in top panel of Fig. 2(a) and (b), respectively.
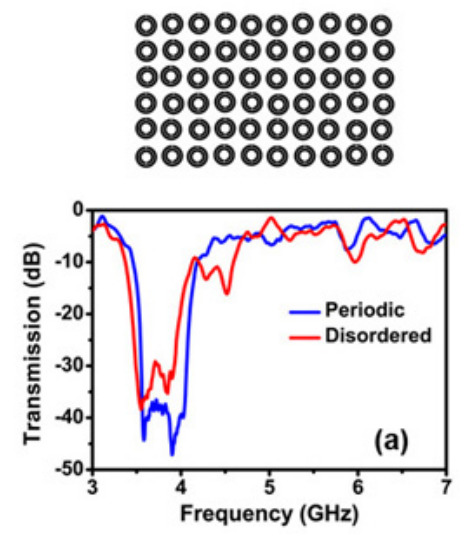
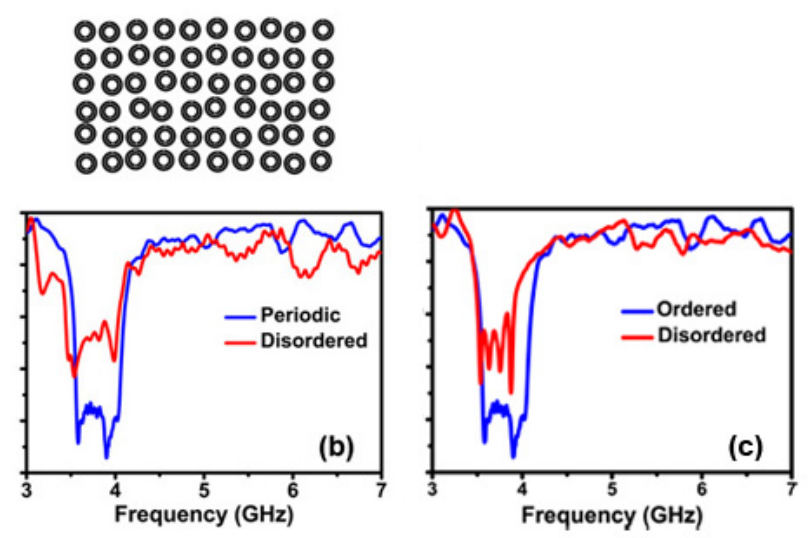

Fig. 2: [Top panel] Schematic drawings of intra-plane disordered SRRs with (a) $\left|\overrightarrow{\boldsymbol{\delta}}_{r}\right| \leq a / 9$, (b) $\left|\overrightarrow{\boldsymbol{\delta}}_{r}\right| \leq a / 5$ where $\overrightarrow{\boldsymbol{\delta}}_{r}$ is the randomness parameter. [Bottom panel] Measured transmission spectra of ordered SRR (blue) and disordered SRR media (red), (c) Comparison of the transmission spectra for ordered and disordered SRRs where the disorder is in all 3 spatial directions.

By introducing disorder, we observe that magnetic resonance band gap still remains, but the transmission inside the band gap is increased with increasing disorder. The average transmission inside the band gap (3.55 - 4.01 GHz) is $40 \mathrm{~dB}$ for ordered structure, $-32 \mathrm{~dB}$ for disordered $\left(\left|\overrightarrow{\boldsymbol{\delta}}_{r}\right| \leq a / 9\right)$ structure (Fig. 2(a)) and $-25 \mathrm{~dB}$ for structure with increased $\left(\left|\overrightarrow{\boldsymbol{\delta}}_{r}\right| \leq a / 5\right)$ disorder (Fig. 2(b)). Each SRR unit has an effect on lowering the transmission and therefore causing a band gap due to magnetic resonance. Adding disorder into SRR structures changes the interaction between the SRRs and this in turn causes a higher transmission inside the band gap. By combining the inter-plane and intraplane disorder, one can obtain a structure, disordered in all three spatial directions. Fig. 2(c) gives the measured transmission spectra for such disordered SRR structure. Magnetic resonance is still present with a relatively higher transmission. A narrower magnetic resonance band gap is observed when compared to ordered SRR structure, and this behavior can be explained by the reduced coupling between SRRs.

\section{References}

[1] V. G. Veselago, "The electrodynamics of substances with simultaneously negative values of permittivity and permeability," Sov. Phys. USPEKHI, 10, 509 (1968).

[2] D. R. Smith, Willie J. Padilla, D. C. Vier, S. C. Nemat-Nasser, and S. Schultz, "Composite medium with simultaneously negative permeability and permittivity," Phys. Rev. Lett., 84, 4184 (2000).

[3] K. Aydin, K. Guven, M. Kafesaki, L. Zhang, C. M. Soukoulis, and E. Ozbay, "Experimental observation of true left-handed transmission peak in metamaterials," Opt. Lett. 29, 2623 (2004). 